\title{
MERS outbreak
}

\section{An outbreak of the respiratory illness MERS in South Korea comes as Muslims are about to embark on their annual pilgrimage to Mecca}

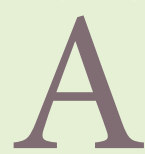

s hundreds of thousands of pilgrims around the world prepare to travel to Saudi Arabia for the hajj in September, many are familiar with Middle East Respiratory Syndrome, or MERS. The illness, known since September 2012, was until recently largely confined to the Arabian peninsula.

But summer 2015 has seen an outbreak of MERS in South Korea, following the return of a businessman after travelling in Saudi Arabia, Bahrain, United Arab Emirates and Qatar: a reminder especially for countries in Southeast Asia that MERS could breach their borders after the hajj.

The businessman was confirmed with MERS on 20 May 2015. In June, over 100 MERS cases appeared in South Korea. As of 12 July, the number of cases stood at 186, with 36 deaths, 130 discharged patients and 20 still under treatment.

This rapid increase sparked concerns about the virus evolving to become more contagious. However, genome sequencing and analysis of virus samples from South Korea have shown this is unlikely. 'The viral genetic sequence did not reveal evidence of changes that relate to altered transmissibility of the virus,' write David Hui, a professor of respiratory medicine at the Chinese University of Hong Kong, and Malik Peiris, a clinical and public health virologist of the University of Hong Kong, in a recent editorial for the American Journal of Respiratory and Critical Care Medicine (doi: 10.1164/rccm.201506-1221ED).

Instead, the South Korean outbreak has been blamed on poor prevention measures, due to a lack of awareness and the unexpectedness of the virus's arrival.
The South Korean businessman did not report his travel history when he first sought medical attention, and then visited three medical facilities before he was diagnosed with MERS over a week later. This 'hospital shopping' is common in South Korea and has been named one of the factors leading to the outbreak.

But this 'happens everywhere in the world,' says Paul Anantharajah Tambyah, an infectious diseases specialist at the National University Hospital in Singapore and SecretaryGeneral of the Asia-Pacific Society of Clinical Microbiology and Infection. 'If a patient doesn't get better, they will look for help elsewhere.'

Overcrowding in medical facilities and a South Korean custom of visiting hospitalised family and friends for long periods of time, even overnight, are likely to have exacerbated the problem. Devices such as nebulisers could also have caused the virus to spread further.

In 2003, a similar illness, Severe Acute Respiratory Syndrome (SARS), terrorised many countries across the globe. Although MERS has a higher mortality rate than SARS, it appears to be less transmissible. In the three years since the first case, there have been 1348 cases globally, according to the World Health Organization. In comparison, SARS affected over 8000 people in six months, says Tambyah, who was among those at the frontlines of Singapore's SARS outbreak.

Since MERS was first identified in 2012, Singapore's Ministry of Health designated a hospital, Tan Tock Seng Hospital, to receive and treat all suspected MERS cases, in order to contain the spread of the illness should it arrive in the country. The same hospital was ground zero of the SARS outbreak in Singapore in 2003. It has received about 180 suspected MERS cases over the last three years; however, none tested positive for the virus.

Several countries in East and Southeast Asia, including Singapore, Hong Kong, Taiwan and Malaysia, have cautioned against travel to South Korea. Tours to South Korea from Hong Kong were cancelled for the month of June 2015 only, disrupting 10,000-20,000 tourists. Singapore began mandatory temperature screening for all air passengers arriving from South Korea in early June.

While there is still no cure for MERS, much research is being done on understanding coronaviruses like MERS and developing treatment options like drugs and vaccines. Currently, MERS patients receive medical care to relieve their symptoms, and to support functioning of vital organs in severe cases. The MERS coronavirus's genome has been sequenced and published, and many in vitro studies on potential drugs and antibodies have been conducted. However, testing these options has been limited because of the relatively small number of MERS cases, says Tambyah; twothirds of South Korea's MERS patients have already recovered and the number of cases has flatlined.

For the annual hajj, Saudi Arabia has implemented several precautions against MERS since 2013, such as strict crowd control, vigilant monitoring and early isolation for suspected cases. In addition, pilgrims from
XiaoZhi Lim is a freelance science writer based in Singapore Thailand and Singapore are required
to receive the influenza vaccination before leaving their countries, so as to make it easier to diagnose MERS. 